\title{
Gait Motion Analysis using Arduino and Blender 3d Software
}

\author{
Nitin Khadane, \\ M.Tech Scholar, Electronics Engineering, JD College of Engineering and Management, Nagpur India \\ Prof. Sunil R Gupta \\ JD College of Engineering and Management, Nagpur India
}

\begin{abstract}
The term 'Gait Analysis' refers to the scientific investigation of animal locomotion, particularly the human locomotion. Determination of gait functionalities, irregularities, and classifications are the main target of gait analysis so that better treatment is possible to provide for non ambulatory patients and also to provide gait enhancement facilities for military or disaster recovery applications. Gait analysis is used to assess and treat individuals with conditions affecting their ability to walk. This paper is intended to do the GAIT analysis of humans. GAIT analysis helps us to predict any problem related to our posture. In this project we will be using Arduino, arduino is an open-source hardware i.e singleboard microcontroller which will be placed on the joints present in the Human body e.g. neck, elbow, shoulder etc. To simulate the movements of the Human body we will do the animations in Blender Software which is completely free of cost and open source 3D computer graphics software toolset .The major focus of this project is to make a low-cost system as this project is intended for Indian citizens who are more conscious of the monetary issues. GAIT analysis using arduino and blender integration is a valid alternative to other expensive techniques.
\end{abstract}

\section{INTRODUCTION}

Human gait is the identity of a person's style and quality of life. Reliable cognition of gait properties over time, continuous monitoring, accuracy of evaluation, and proper analysis of human gait characteristics have demonstrated their importance not only in clinical and medical studies, but also in the field of sports, rehabilitation, training, and robotics research.[1] Focusing on walking gait, this study presents an overview on gait mechanisms, common technologies used in gait analysis, and importance of this particular field of research[3]. The paper also shows the importance of gait analysis in sports to improve performance as well as to avoid risk of injuries of sports personnel. Significance of gait analysis in robotic research is also illustrated where the study focuses on robot assisted systems and its possible applicability in clinical rehabilitation and sports training.

\section{GAIT Analysis}

Gait analysis is a very important component of competitive and recreational running performance. The study encompasses quantification i.e. introduction and analysis of measurable parameters of gaits, as well as interpretation, i.e. drawing various conclusions about the human (health, age, size, weight, speed etc.) from its gait pattern[8].

\section{Factors and parameters}

The gait analysis is modulated or modified by many factors, and changes in the normal gait pattern can be transient or permanent. The factors can be of various types[9]:

- Extrinsic: such as terrain, footwear, clothing, cargo

- Intrinsic: sex (male or female), weight, height, age, etc.

- Physical: such as weight, height, physique

- Psychological: personality type, emotions

- Physiological: anthropometric characteristics, i.e., measurements and proportions of body

- Pathological: for example trauma, neurological diseases, musculoskeletal anomalies, psychiatric disorders

\section{MOTIVATION/PROBLEM STATEMENT}

Gait analysis is a very important component of competitive and recreational running performance. However it is often a last option when analyzing performance, and completed only after injury has taken place. Preventative gait analysis should be a critical component of performance analysis, as it could be used to optimize form, efficiency, energy expenditure,and to potentially decrease injury risk. The problem is that gait analysis requires expertise, expensive equipment, and a large amount of time. This is a matter of great inconvenience for the runner with no visible injury or noticeable problem with technique, and is the main reason gait analysis is often used only to correct injury, and not preventatively. Gait analysis provides critical detail needed to prevent injury in athletes. Proper mechanics will also increase performance by increasing running efficiency. [6]Mechanics are key to success in all distances of running events, though improper mechanics during long distance runs will have an increasingly detrimental effect as the runner continues to train and stress their joints. Proper mechanics and technique will also lead to increased strength and power through achievement of bilateral equivalence[7]. A properly designed strength and conditioning program can be developed from data that can be provided by blender integration using arduino. Gait 
analysis is an important component of analyzing running performance. It is necessary, and will aid in determining how to correct mechanics to optimize running efficiency/economy, and to prevent injury and prolong career.Gait analysis is expensive and not practical, and is often an overlooked variable that could help increase performance.

\section{PROPOSED SYSTEM}

\subsection{Arduino:-}

Arduino is an open-source software. Arduino board designs use a variety of microprocessors and controllers. The boards are equipped with sets of digital and analog input/output (I/O) pins that may be interfaced to various expansion boards. Arduino IDE supports the languages $\mathrm{C}$ and $\mathrm{C}++$ using special rules of code structuring.[5] The Arduino IDE supplies a software library from the Wiring project, which provides many common input and output procedures. User-written code only requires two basic functions, for starting the sketch and the main program loop, that are compiled and linked with a program stub main() into an executable cyclic executive program with the GNU tool chain, also included with the IDE distribution[8].

\subsection{Blender Inegration Software:-}

Blender is a professional, free and open-source 3D computer graphics software toolset used for creating animated films, visual effects, art, 3D printed models, interactive 3D applications[10].

Blender is used for Microsoft Windows, MacOS and Linux, as well as a port for Free $\mathrm{BSD}$, are available in both 32-bit and 64-bit versions.

- $\quad$ Simulation tools for soft body dynamics including mesh collision detection, LBM fluid dynamics, smoke simulation, Bulletrigid body dynamics, ocean generator with waves.

- Particle system that includes support for particlebased hair.

- Modifiers to apply non-destructive effects.

- Python scripting for tool creation and prototyping, game logic, importing/exporting from other formats, task automation and custom tools.

- Basic non-linear video/audio editing.

- A fully integrated node-based compositor within the rendering pipeline accelerated with Open CL.

- Procedural and node-based textures, as well as texture painting, projective painting, vertex painting, weight painting and dynamic painting.

- Real-time control during physics simulation and rendering.

\section{Result}

GAIT analysis arduino and blender integration is a valid alternative to other expensive techniques. It provides an effective technique for real time motion capture, It provides a higher range and flexibility of movement and usage in outdoor environments.

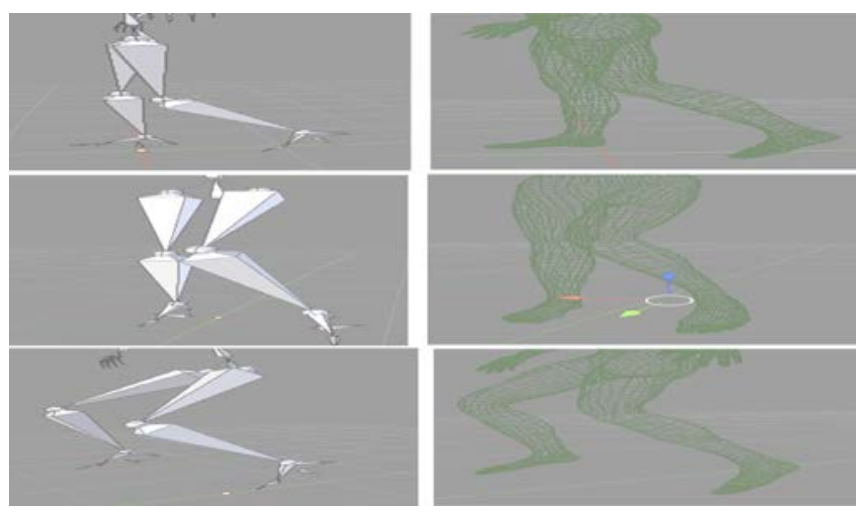

Fig 1. Result of different leg positions

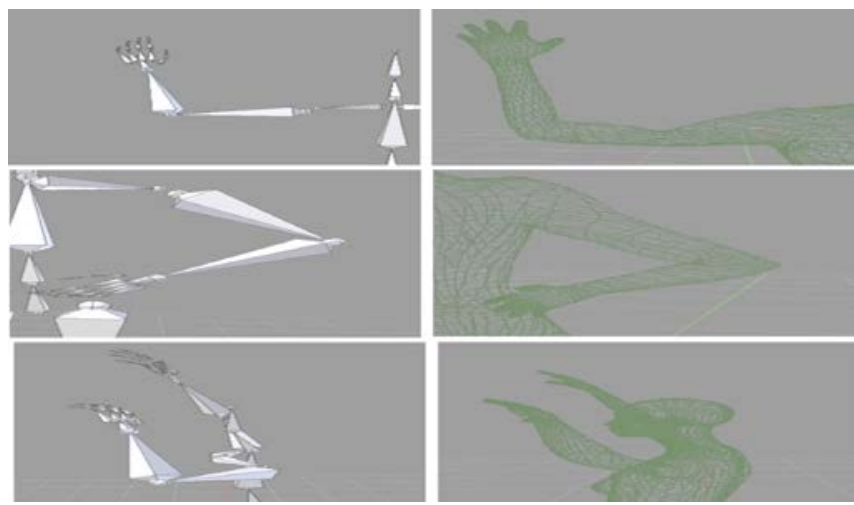

Fig 2. Result of arm,body leg positions

\section{CONCLUSION}

Gait analysis using arduino and blender integration is a product that can bring gait analysis to runners without the need for expensive, time-consuming laboratory equipment that requires an expert in order to test and analyze. It enhance running performance and potentially prevent injury. Running performance is improved through gait analysis feedback.

\section{REFERENCES}

[1] Davis, R.B., Õunpuu, S., Tyburski, D., Gage, J.R.: A gait analysis data collection and reduction technique. Hum. Mov. Sci. 10, 575587 (1991)

[2] H. Weiming, T. Tieniu , W. Liang, and S.Maybank, "A survey on visual surveillance of object motion and behaviours," IEEE Transactions on Systems, Man, and Cybernetics, Applications and Reviews., vol.34, no. 3, pp. 334-352, 2004.

[3] J. Robert, and G. D. Abowd. "The smart floor: a mechanism for natural user identification and tracking,"CHl'00 extended abstracts on human factors in computing systems., pp. 275276, 2000.

[4] M. Gleicher, "Animation from observation: Motion capture and motion editing,” ACM SIGGRAPH Computer Graphics, vol. 33, no. 4, pp. 51- 54, 1999.

[5] D. J. Sturman, “A Brief History of Motion Capture for Compute Char- acter Animation,” ACM SIGGRAPH '94, vol. Cours Notes: Character Animation Systems, 1994. 
[6] M. Field, D. Stirling, F. Naghdy, and Z. Pan, "Motion capture in robotics review," in Control and Automation, 2009. ICCA 2009. IEEE International Conference on, pp. 1697-1702, IEEE, 2009.

[7] W. Li, Z. Zhang, and Z. Liu, "Expandable data-driven graphical model- ing of human actions based on salient postures," IEEE transactions on Circuits and Systems for Video Technology, vol. 18, no. 11, pp. 1499- 1510, 2008.

[8] N. C. Perkins, "Electronic measurement of the motion of a moving body of sports equipment,” June 26 2007. US Patent 7,234,351.

[9] D. Vlasic, R. Adelsberger, G. Vannucci, J. Barnwell, M. Gross, W. Ma- tusik, and J. Popović, "Practical motion capture in everyday surround- ings," in ACM transactions on graphics (TOG), vol. 26, p. 35, Acm, 2007.

[10] M. Loper, N. Mahmood, and M. J. Black, "Mosh: Motion and shape capture from sparse markers," ACM Transactions on Graphics

\begin{abstract}
vol. 33 ,
\end{abstract}
2014

(TOG),
, no.

\begin{abstract}
6, p. 220,
\end{abstract}

